\title{
LAS ARMAS DE DESTRUCCION MASIVA Y LA ESTRATEGIA GLOBAL DE SEGURIDAD DE LA UNIÓN EUROPEA
}

\author{
Mónica Miranzo ${ }^{1}$ \\ UNISCI
}

\begin{abstract}
Resumen:
La proliferación de Armas de Destrucción Masiva (ADM), Nucleares, Biológicas y Químicas (NBQ) es una de las principales amenazas a la seguridad que enfrenta la comunidad internacional en la actualidad Sin embardo la nueva Estrategia Global de Seguridad de 2016 plantea la cuestión de la no proliferación de ADM sólo de forma tangencial, sin abordar la amenaza directamente, un problema fundamental en la seguridad. Esto supone un claro paso atrás para la seguridad común europea, que ha optado por una solución de mínimos que pone fin a los avances relativos que se habían realizado en la materia.
\end{abstract}

Palabras clave: Armas de Destrucción Masiva, No Proliferación, Tratado de no proliferación nuclear, Conferencias de Revisión, Estrategia Global de Seguridad de la UE.

Title in English: The Weapons of Mass Destruction and the Global Security Strategy of the EU

\begin{abstract}
:
The proliferation of weapons of mass destruction (WMD), nuclear, biological and chemical $(N B C)$ is one of the main security challenges facing the international community today. However the new Global Security Strategy of 2016 raises the question of non-proliferation of WMD only as an incidental matter, not addressing directly the threat, a fundamental threat in the regional and global security. This is a clear step backwards for the European common security.
\end{abstract}

Keywords Weapons of Mass Destruction, Non-proliferation, Nuclear Non-proliferation Treaty, NPT review Conferences, Global Security Strategy of the EU.

Copyright (C) UNISCI, 2016.

Las opiniones expresadas en estos artículos son propias de sus autores, y no reflejan necesariamente la opinión de UNISCI. The views expressed in these articles are those of the authors, and do not necessarily reflect the views of UNISCI.

\footnotetext{
${ }^{1}$ Monica Miranzo es investigadora de UNISCI

Email: mmiranzop@yahoo.es

DOI: http://dx.doi.org/10.5209/RUNI.53790
} 


\section{Introducción.}

La proliferación de Armas de Destrucción Masiva (ADM), Nucleares, Biológicas y Químicas (NBQ) es una de las principales amenazas a la seguridad que enfrenta la comunidad internacional en la actualidad. La Organización de Naciones Unidas (ONU) ya reconocía en una declaración emitida por presidente del Consejo de Seguridad en 1992 que "la proliferación de armas de destrucción masiva constituye una amenaza para la paz y la seguridad internacionales" ${ }^{2}$ e instaba a los Estados Miembros a prevenir la proliferación de Armas de Destrucción Masiva mediante la implementación de programas de desarme, el control de los arsenales y la prevención de las transferencias tecnológicas ${ }^{3}$.

A comienzos del S. XXI la amenaza potencial del uso de Armas de Destrucción Masiva ha alcanzado una nueva dimensión, en función del desarrollo, por parte de nuevos Estados, de arsenales NBQ y de la posibilidad de adquisición de estos arsenales por parte de actores de carácter no estatal, especialmente grupos terroristas ${ }^{4}$ a partir del año 2001.

En este escenario, la Unión Europea no ha permanecido indiferente al problema de la proliferación. Durante la Primera Sesión del Comité Preparatorio para la Conferencia de Revisión de 2005 del Tratado de No Proliferación de Armas Nucleares (TNP), celebrada en Nueva York en el año 2002, el embajador-delegado de España en representación de la Unión Europea y de los países asociados, Carlos Miranda, dejaba patente que "Los acontecimientos del 11 de septiembre han imprimido un sentimiento, si cabe aun mayor, de urgencia a los esfuerzos comunes exigibles a todos los Estados para combatir la amenaza que representa para la seguridad y estabilidad internacionales el acceso de los grupos terroristas a las armas de destrucción masiva".

La salida de Corea del Norte del Tratado de No Proliferación en 2003, unida a la necesidad de superar las divisiones internas propiciadas por la guerra de Irak, llevaron a la Unión a consolidar una posición común en cuestiones de seguridad, con una visión estratégica más amplia y una clara priorización de las políticas de lucha contra el terrorismo y la proliferación de $\mathrm{ADM}^{6}$, mediante la aprobación de la Estrategia Europea de Seguridad "Una Europa segura en un mundo mejor" durante el Consejo de Salónica y de la adopción de la Estrategia contra la Proliferación de Armas de Destrucción Masiva del año 2003.

En este artículo nos centramos en la proliferación nuclear, si bien hay que subrayar la importancia creciente que tiene la proliferación de armas químicas- que se han utilizado en varias ocasiones en la guerra en Siria- y, de forma especial, las armas biológicas, las armas del siglo XXI. Sorprendentemente se ha pasado de afirmar en la Estrategia Europea de Seguridad (EES) de 2003 que: "Los progresos de las ciencias biológicas pueden aumentar la potencia de las armas biológicas en los próximos años; los atentados con sustancias químicas y radiológicas también son una posibilidad verosímil", a una reducción en el Informe de

\footnotetext{
${ }^{2}$ Declaración del presidente del Consejo de Seguridad de la ONU de 31 de enero de 1992, S/23500, en http://www.securitycouncilreport.org/un-documents/document/PKO\%20S\%2023500.php, p. 3

3 Ibid, p. 4.

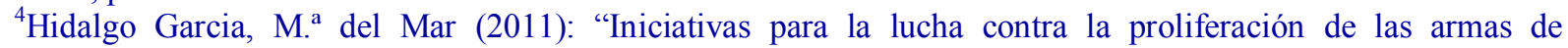
destrucción masiva”, en Proliferación de ADM y de tecnología avanzada, Ministerio de Defensa. Cuadernos de estrategia $\mathrm{n}^{\mathrm{o}}$ 153. IEEE (septiembre 2011), p. 15.

5 Garrido Rebolledo, Vicente: "Redefinición y respuesta de los europeos frente a las nuevas amenazas: proliferación de armamento de destrucción masiva”, en La seguridad europea y las incertidumbres del 11 de septiembre, Monografías del CESEDEN nº 61, mayo 2003, p. 79.

6 Vazquez Muñoz, Manuel: "La respuesta europea ante la amenaza de la proliferación de las armas de destrucción masiva", UNISCI Discussion Papers, (mayo 2004), p. 2.
} 
aplicación de la Estrategia Europea de Seguridad en 2008, donde meramente se habla de medidas de bioprotección y bioseguridad, y a su disolución temática en la Estrategia Global de Seguridad de 2016, incluyéndolas, sin citarlas, en el apoyo de la UE a los regímenes y tratados de no proliferación.

\section{Antecedentes inmediatos. El liderazgo estadounidense en el control de la proliferación.}

La aprobación por parte del presidente George Bush de la Estrategia de Seguridad Nacional para combatir las ADM (ESN) el 11 de diciembre de 2002 y la promoción de la Iniciativa de Seguridad contra la Proliferación (PSI) el 31 de mayo de 2003, constatan el liderazgo estadounidense en materia de control de la proliferación "que abre la puerta a una toma de postura internacional ante las $\mathrm{ADM}^{\text {"7 }}$ y resulta en la aprobación Resolución 1540 por el Consejo de Seguridad de Naciones Unidas el 28 de abril de $2004^{8}$.

La ESN identifica como principal amenaza a la seguridad estadounidense e internacional la adquisición de ADM por parte de grupos terroristas y de "rogue states" y asienta sus líneas de acción en este ámbito sobre tres ejes fundamentales: la contraproliferación para combatir el uso de ADM, el reforzamiento de la no proliferación y la gestión consecuente para responder al uso de $\mathrm{ADM}^{9}$; poniendo de manifiesto la opción estadounidense por el unilateralismo de carácter preventivo: “(...)EEUU se esforzará constantemente por conseguir el apoyo de la comunidad internacional, no dudaremos para actuar aisladamente si es necesario, para ejercer nuestro derecho de autodefensa actuando preventivamente contra terroristas, evitando que hagan daño a nuestro pueblo y a nuestro país... EEUU no puede continuar con la posición reactiva que teníamos en el pasado". ${ }^{10}$

La adopción de la PSI y los principios de interdicción supone la materialización de las ambiciones de contraproliferación estadounidenses, con un amplio respaldo europeo e internacional $^{11}$, mediante la realización de operaciones de bloqueo marítimas, aéreas y terrestres para evitar la transferencia de ADM, sus sistemas vectores y los materiales relacionados desde y hacia los Estados y actores no estatales que son motivo de inquietud por posibilidades de proliferación ${ }^{12}$. Llegando incluso a detener, registrar, e incluso confiscar, cargamento de este tipo de los buques y aeronaves que se encuentren en su espacio aéreo o en sus aguas interiores, mar territorial o zonas contiguas, con destino u origen de Estados o actores no estatales que son motivo de inquietud por posibilidades de proliferación, cuando exista una sospecha razonable de transporte sobre su carga, así como la denegación de los derechos de tránsito ${ }^{13}$.

\footnotetext{
${ }^{7}$ Guisández Gómez, Javier: "Iniciativas multilaterales de no proliferación", en Respuestas al reto de la proliferación, Documentos de Seguridad y Defensa, CESEDEN, nº 27, marzo 2009, p. 36.

${ }^{8}$ La Resolucion 1540 establece que "todos los Estados, de conformidad con sus procedimientos, deben adoptar y aplicar leyes apropiadas y eficaces que prohíban a todos los agentes no estatales la fabricación, la adquisición, la posesión, el desarrollo, el transporte, la transferencia o el empleo de armas nucleares, químicas o biológicas y sus sistemas de vectores, en particular con fines de terrorismo, así como las tentativas de realizar cualquiera de las actividades antes mencionadas, participar en ellas en calidad de cómplices, prestarles asistencia o financiarlas".

${ }^{9}$ The National Security Strategy of the United Status of America (NSS). Washington D.C., September 2002, en http://www.whitehouse.gov/nsc/nss.html.

${ }^{10}$ Garrido Rebolledo, op. cit. p. 79.

${ }^{11}$ Los 11 países fundadores de la PSI son Francia, Alemania, Australia, España, Estados Unidos, Italia, Japón, Países Bajos, Polonia, Portugal y Reino Unido.

${ }^{12}$ Proliferation Security Initiative: Statement of Interdiction Principles, en http://www.psionline.info/Vertretung/psi/en/07-statement/Interdiction-Principes.html

13 Ibid.
} 
Por lo tanto, las acciones implementadas por EEUU son el principal exponente de una política de contraproliferación, basada en el uso de la fuerza, o la amenaza de esta, de forma directa o mediante el despliegue del sistema de defensa antimisiles (NMD) ${ }^{14}$, de forma unilateral y con carácter discriminatorio en función de dos objetivos fundamentales: los Estados denominados proliferadores, en el caso de Corea del Norte e Irán, y los Estados facilitadores involuntarios o "leakers", como Rusia o Pakistán, cuya falta de control puede generar fugas de estas tecnologías ${ }^{15}$.

\section{La consolidación de la política de no proliferación europea: la Estrategia Europea de Seguridad y la Estrategia contra la proliferación de armas de destrucción masiva y sus vectores.}

En el caso europeo, la PSI supuso la medida de referencia en la adopción de la Estrategia Europea de Seguridad (EES) y la Estrategia contra la proliferación de armas de destrucción masiva y sus vectores, ambas aprobadas en el año 2003 y que presentarían diferencias sustanciales con la perspectiva estadounidense, presentándose como principal alternativa al unilateralismo norteamericano.

La EES se asienta sobre la constatación de que ningún país es capaz de afrontar los retos de la seguridad de forma individual, las nuevas amenazas no presentan un carácter "meramente militar, ni pueden atajarse únicamente con medios militares"16. La creación de un entorno de seguridad europeo como objetivo último de la EES se basa en la doble premisa de que en "un mundo de amenazas, mercados y medios de comunicación globales, nuestra seguridad y nuestra prosperidad dependen cada vez más de la existencia de un sistema multilateral eficaz"17, esto implicaría "el desarrollo de una sociedad internacional más fuerte, con instituciones internacionales que funcionen adecuadamente, y de un orden internacional basado en el Derecho ${ }^{18}$.

En este contexto, la EES identifica la proliferación de armas de destrucción masiva como una de las principales amenazas a la seguridad de la Unión Europea, junto al terrorismo, los conflictos regionales, la descomposición del Estado y la delincuencia organizada. La proliferación de ADM es identificada, de hecho, como la amenaza potencial más grave a la seguridad europea ${ }^{19}$, especialmente en función de tres elementos clave que condicionan el problema:

- la posibilidad de una carrera armamentística en Oriente Próximo,

- la expansión de los arsenales biológicos en función del desarrollo científico y tecnológico de los países

- $\quad y$, en última instancia, la adquisición de $\mathrm{ADM}$ por parte de los grupos terroristas.

En relación a este último punto, la EES sostiene que "la adquisición de armas de destrucción masiva por grupos terroristas constituye el escenario más temible. Si se produjera,

\footnotetext{
${ }^{14}$ En la obra citada en la nota 4 se explica cómo la opción de los EEUU por medidas de control de la proliferación principalmente militares, especialmente el NMD han contribuido directamente a deteriorar algunos de los logros alcanzados en materia de no proliferación especialmente en la medida en que los instrumentos adoptados por los EEUU se basan "exclusivamente en la respuesta militar al problema, aun a costa de sacrificar los tratados existentes, como el ABM o el CTBT".

${ }^{15}$ Nia, Mahdi Mohammad: "Counter-proliferation vs Non-proliferation.", PA Pundits-International, 2008, en https://papundits.wordpress.com/2008/11/20/counter-proliferation-vs-non-proliferation/

${ }_{16}$ A Secure Europe in a Better World, p. 8 en https://www.consilium.europa.eu/uedocs/cmsUpload/78367.pdf

${ }^{17}$ Ibid., p. 9.

${ }^{18}$ Ibid., p. 9.

${ }^{19}$ Ibid., p. 3.
} 
un grupo pequeño podría causar daños de una magnitud que antes sólo estaba al alcance de los Estados y los ejércitos" ${ }^{20}$.

Paralelamente a esta declaración de principios, el 12 de diciembre de 2003, el Consejo Europeo adoptó la Estrategia de la UE contra la proliferación de armas de destrucción masiva y sus vectores, que viene a concretar la acción de la Unión en este ámbito, optando exclusivamente por la utilización de la diplomacia preventiva y la cooperación como instrumento clave para "prevenir, disuadir, detener y, si es posible, eliminar los programas de proliferación de ADM que preocupan a nivel mundial" 21 . La defensa de estos objetivos recae en los siguientes principios básicos:

- Reforzar los mecanismos internacionales de no proliferación y trabajar para mejorar los sistemas de verificación de las violaciones de las normas dispuestas en tratados multilaterales ${ }^{22}$.

- Fomentar un entorno estable a escala regional e internacional a través del refuerzo de los programas que promuevan el desarme y de la incorporación del objetivo de no proliferación a todas las actividades políticas, diplomáticas y económicas de la UE23.

- Establecer una estrecha cooperación con países asociados de mayor importancia como los Estados Unidos, Rusia o los países de la OTAN y asistir a países de fuera de la $\mathrm{UE}^{24}$.

- En última instancia se abriría la puerta a la utilización de medidas coercitivas previstas en el capítulo 7 de la Carta de las Naciones Unidas, incluyendo sanciones selectivas o globales, interceptación de cargamentos y, en su caso, el uso de la fuerza ${ }^{25}$.

La opción europea por las herramientas de "soft-power", incluyendo medidas coercitivas, que estaban articuladas sobre tres ejes principales: la diplomacia, la disuasión y la contención, ha definido una estrategia de objetivos limitados, con una clara falta de priorización y un notable grado de ambigüedad. La estrategia en ningún caso contempla la desaparición de las ADM y solo considera su existencia como amenaza en la medida en que puedan ser adquiridas por Estados proliferadores o por grupos terroristas, sin llegar a concretar los criterios de definición de un programa de proliferación ${ }^{26}$.

El empeoramiento del régimen de no proliferación ${ }^{27}$ durante estos años, propiciado por el distanciamiento de Rusia y EEUU, después de la retirada unilateral de este último del Tratado ABM, respondida con la retirada rusa del Tratado START II (después sustituido por un diluido Tratado SORT), y por la aparición de dos Estados proliferadores, Irán y Corea del

\footnotetext{
${ }^{20}$ Ibid., p. 4.

${ }^{21}$ Estrategia de la UE contra la proliferación de armas de destrucción masiva, p. 8, en http://register.consilium.europa.eu/doc/srv?l=ES\&f=ST\%2015708\%202003\%20INIT

${ }^{22}$ Armas de destrucción masiva: lucha contra la proliferación, en http://eur-lex.europa.eu/legalcontent/ES/TXT/?uri=uriserv\%3Al33234

${ }^{23}$ Ibid.

${ }^{24}$ Ibid.

${ }^{25}$ Estrategia de la UE contra la proliferación de armas de destrucción masiva, p. 5, en http://register.consilium.europa.eu/doc/srv?l=ES\&f=ST\%2015708\%202003\%20INIT

26 Álvarez, Milagros: "La Aproximación de las Estrategias de la UE y de los EEUU en la lucha contra la proliferación de armas de destrucción masiva”, UNISCI Discussion Papers, no 30 (octubre 2012) p. 47.

${ }^{27}$ El Informe del Grupo de Alto Nivel sobre las amenazas, los desafíos y el cambio "Un mundo más seguro: la responsabilidad que compartimos" elaborado por Naciones Unidas en el año 2004 llegaba a sostener que "estamos llegando a un punto en el que el debilitamiento del régimen de no proliferación podría llegar a ser irreversible y provocar una proliferación en cascada".
} 
Norte, enfrenta al mundo a una "segunda era nuclear" que se inicia en 2007 y propicia una evaluación de la política de no proliferación europea ${ }^{28}$.

En este nuevo contexto, el 11 de diciembre de 2008 se presentó el Informe sobre la aplicación de la Estrategia Europea de Seguridad "Ofrecer seguridad en un mundo en evolución", que muestra las nuevas líneas de actuación de la Unión Europea elaboradas para mejorar la eficacia de la estrategia en la lucha contra la proliferación de armas de destrucción masiva y sus vectores ${ }^{29}$. El informe está encaminado a desarrollar los siguientes aspectos ${ }^{30}$ :

- Intensificar los esfuerzos para contrarrestar los flujos de proliferación y financiación de la proliferación.

- Sancionar los actos de proliferación.

- Desarrollar medidas para prevenir las transferencias intangibles de conocimiento y know how.

- Sensibilizar a las empresas, los círculos científicos y académicos y las instituciones financieras.

- Continuar la cooperación con las organizaciones internacionales y terceros países para ayudarles a mejorar las políticas de no proliferación y control de las exportaciones.

El informe de 2008 no pretende suplantar la EES del año 2003 sino adaptarla a un nuevo contexto de seguridad en función de los cambios que se han producido en la sociedad internacional. La proliferación de ADM continúa siendo un asunto de importancia capital para la Unión, que se muestra especialmente sensibilizada ante la amenaza que suponía el desarrollo del programa nuclear iraní, que llega a ser definido como "una amenaza para la seguridad de la UE que no podemos aceptar"31. Esta declaración, sin embargo, queda atemperada por la falta de concreción de las medidas de respuesta "Si en lugar de ello siguiera adelante el programa nuclear, aumentaría la necesidad de medidas complementarias en apoyo del proceso de la ONU"32.

En este sentido, el Informe sigue de forma inercial los planteamientos ya enunciados en la EES del año 2003 y se asienta sobre el enunciado de líneas de acción generales que presentan muy pocas variaciones respecto a la situación anterior. Sin embargo, dos son los aspectos que merecen ser tenidos en cuenta: por una parte, la toma de conciencia de la necesidad de alejarse de un enfoque global dentro de la problemática de la proliferación, descendiendo al análisis de caso en función de su impacto sobre la seguridad de la Unión ${ }^{33} \mathrm{y}$,

\footnotetext{
${ }^{28}$ La falta de avances en las iniciativas de control de la proliferación durante estos años y el enfriamiento internacional había generado un estancamiento en las Conferencias de revisión del TNP, llegando a finalizar la del año 2005 sin acordar un plan de acción para afrontar el desarme nuclear y la no proliferación.

${ }^{29}$ El Informe de evaluación de la aplicación se complementa con la aprobación del documento: "Nuevas líneas de acción de la UE en el combate contra la proliferación de ADM y sus sistemas vectores". El 8 de diciembre de 2008, el Consejo había adoptado este documento donde se enfatiza que las armas de destrucción masiva que pudieran caer en manos de Estados de especial preocupación o terroristas/ actores no estatales constituían una de los mayores desafíos a los que los europeos debían hacer frente. El objetivo era mejorar la puesta en práctica de la Estrategia contra la proliferación y hacer de la no proliferación una prioridad transversal en las políticas de los Estados Miembros

${ }^{30}$ Council Conclusions and new lines for action by the European Union in combating the proliferation of weapons of mass destruction and their delivery systems. European Union External Action The fight against proliferation of WMD, en http://eeas.europa.eu/non-proliferation-and-disarmament/wmd/index en.htm

${ }^{31}$ Report on the Implementation of the European Security Strategy - Providing Security in a Changing World-, p.

7, en https://www.consilium.europa.eu/ueDocs/cms_Data/docs/pressdata/EN/reports/104630.pdf

${ }^{32}$ Ibid.

${ }^{33}$ Álvarez, op. cit., p. 56.
} 
por otra, el impulso en la consolidación de medidas de control de exportaciones y tecnologías de doble uso ${ }^{34}$.

\section{Los límites internos del sistema de no proliferación europeo y el engarce trasatlántico.} Si bien la adopción de la EES y la Estrategia contra la proliferación de ADM ha plasmado la resolución tentativa de la Unión Europea en dotarse de voz propia respecto a una de las cuestiones fundamentales que afectan a la seguridad internacional tratando de actuar de manera coordinada en materia de ADM, también ha puesto de manifiesto las limitaciones de la Unión en el logro del consenso en función de dos elementos condicionantes: el primero es la existencia de dos Estados miembros, Francia y Reino Unido, con armamento nuclear en el seno de la UE, y el segundo es el vínculo transatlántico como elemento fracturador de una política de seguridad común ${ }^{35}$.

La llegada de Barack Obama a la Presidencia de los EEUU marcó un cambio de tendencia con la adopción, en el año 2009, de la "National Strategy for Countering Biological Threats" y la adopción de la "Nuclear Posture Review" (NPR) y la "National Security Strategy" en 2010. Estos documentos constatan la adopción de una nueva filosofía en la política de control de la proliferación estadounidense, materializada en el discurso del Presidente Obama en Praga el 5 de abril de 2009, donde expone que: "La existencia de miles de armas nucleares es el legado más peligroso de la Guerra Fría (...) Hoy, miles de esas armas todavía existen. En un raro giro de la historia, la amenaza de una guerra nuclear ha disminuido pero el riesgo de un ataque nuclear se ha incrementado. (...)Y como potencia nuclear, como la única potencia que ha utilizado un arma nuclear, los Estados Unidos tienen la responsabilidad moral de actuar. (...) Entonces hoy, afirmo claramente y con convicción el compromiso de América de buscar la paz y la seguridad de un mundo sin armas nucleares"36. Los cambios planteados por la administración Obama en materia de ADM se materializan en cuatro ámbitos: el fortalecimiento de los sistemas de control para evitar que los grupos terroristas adquieran ADM; el reseteo de las relaciones con Rusia y la promoción de medidas de reducción de arsenales y desarme a través de la entrada en vigor de un nuevo Tratado START que reduce en un $30 \%$ más sus arsenales ${ }^{37}$; el impulso de los mecanismos de no proliferación y verificación internacionales sobre la base del TNP y la aprobación de un Plan de Acción en el año 2010 para negociar el establecimiento en Oriente Medio de una zona libre de armas nucleares y de otras armas de destrucción masiva ${ }^{38}$; y por último, la implantación de un nuevo proyecto antimisiles en Europa, el European Phased Adaptive Approach (EPAA) ${ }^{39}$ un sistema flexible ampliamente aceptado por los países europeos y menos controvertido y amenazante para Rusia ${ }^{40}$.

Esta "relajación" del tradicional unilateralismo estadounidense en función de la dilución del uso de la fuerza propició un acercamiento doctrinal a la posición europea en

\footnotetext{
${ }^{34}$ Portela, Clara: "The Role of the EU in the Non-Proliferation of Nuclear Weapons: The Way to Thessaloniki and Beyond", Peace Research Institute Frankfurt, PRIF Reports, nº6 (2003), p. 29.

${ }^{35}$ Ibid., pp. 3-4.

${ }^{36}$ Discurso del Presidente de los Estados Unidos, Barack Obama en Praga. 5 abril 2009, en http://npsglobal.org/esp/discursos/574-discurso-de-barack-obama-en-praga.html

${ }^{37}$ Salazar, Gonzalo de: "El tratado de no proliferación de armas nucleares: los temas clave en la conferencia de examen en 2015", UNISCI Discussion Papers, n 38 (mayo 2015), p. 158.

${ }^{38}$ NPT RevCon Final Document- NPT/CONF.2010/50, en Www.kcl.ac.uk/sspp/departments/warstudies/research/groups/csss/pubs/bb2010.pdf

${ }^{39}$ Fact Sheet on U.S. Missile Defense Policy. A Phased, Adaptive Approach for Missile Defense in Europe, 17 Sept. 2009), en https://www.whitehouse.gov/the-press-office/fact-sheet-us-missile-defense-policy-a-phasedadaptive-approach-missile-defense-eur

${ }^{40}$ Lara, Belén: "Europa y las defensas antimisiles", UNISCI Discussion Papers, no 30 (octubre 2012), pp. 93-96.
} 
materia de no proliferación. En sentido inverso, la nueva postura estadounidense favoreció la revisión de las iniciativas de desarme de Francia y Reino Unido, que se han mostrado dispuestos, especialmente a partir de 2010, a propiciar una reducción de sus arsenales nucleares.

Sin embargo, en la práctica, en el caso de Rusia y EE.UU. esta reducción de los arsenales nucleares ha respondido a planteamientos pragmáticos y ha sido compaginada con las mejoras cualitativas derivadas de la implantación de programas de modernización ${ }^{41}$. En el caso de Francia, la reducción de su arsenal nuclear se detuvo en el año 2008, estabilizándose en 300 cabezas nucleares desplegadas, si bien el gobierno francés se ha comprometido a no superar este límite, en el año 2013 inició un programa de modernización con un coste de 180.000 millones de euros con horizonte $2019^{42}$. El Reino Unido también ha propiciado una reducción paulatina de su arsenal nuclear que cuenta con 215 cabezas nucleares en la actualidad y que serán reducidas a 180 en la década de $2020^{43}$. Siguiendo la dinámica global de modernización, el Parlamento británico aprobó en julio de 2016 una actualización y mejora del sistema nuclear Trident con un coste estimado de 40.000 millones de dólares.

En cualquier caso, las contradicciones existentes en el seno de la EU habían hecho su aparición en las conferencias de revisión del TNP, de forma especial entre los Estados Miembros que han mantenido una tradicional posición de neutralidad y los Estados con armas nucleares. La UE no podrá tener un papel de liderazgo, también porque no podía entrar en contradicción con los Estados Unidos, y tendrá una capacidad de negociación limitada, si bien previamente a las Conferencias de revisión, adoptará posiciones comunes y enfatizará la observancia y el refuerzo de los tres pilares: desarme, no proliferación y uso pacífico de la energía nuclear en la revisión del TNP. No obstante, con respecto al desarme nuclear, Francia creó problemas en la Conferencia de revisión de 2005 al alinearse de alguna manera con Estados Unidos en la no aceptación de los resultados de la Conferencia de revisión de 2000 como base de la negociación, en particular los 13 pasos prácticos sobre desarme nuclear que afectando al artículo 6 del tratado, le convertían en un tratado más equilibrado. También el uso pacífico de la energía nuclear ha planteado problemas entre los Estados Miembros. ${ }^{44} \mathrm{En}$ cualquier caso, en la Conferencia de revisión de 2010, dado el cambio de actitud de los Estados Unidos con respecto al desarme, con la nueva administración Obama, e incluso la participación del Servicio Exterior de la UE, tras el tratado de Lisboa, la UE pudo jugar esta vez un papel menos ambiguo que en anteriores ocasiones ${ }^{45}$ y se conseguirá adoptar un plan de acción, el primero en la historia del tratado.

Hay que tener cuenta también que la disuasión nuclear constituye un elemento central en la política de seguridad de Francia y Reino Unido de forma explícita, y de los Estados Miembros que son, a su vez miembros de la OTAN, cuyo concepto estratégico aprobado en Lisboa el 19 de noviembre de 2010 establece que "La disuasión, articulada en torno a una combinación apropiada de capacidades nucleares y convencionales, son un elemento central en el conjunto de nuestra estrategia. Las condiciones en las que se podría recurrir al arma

\footnotetext{
${ }^{41}$ Miasnikov, Eugene: "Modernizing nuclear arsenals: Whether and how" y "Modernizing and "zero": compatible tendencies?", Bulletin of Atomic Scientists, (enero 2015), en http://thebulletin.org/modernizingnuclear-arsenals-whether-and-how7881

${ }^{42}$ NTI. France, en http://www.nti.org/learn/countries/france/nuclear/

${ }^{43}$ NTI. United Kingdom, en http://www.nti.org/learn/countries/united-kingdom/

${ }^{44}$ Dee Megan: "Explaining European Union performance in the nuclear non-proliferation treaty review Conference: Limited ambitions but pragmatic positioning”, UNISCI Discussion Papers, nº 30 (Octubre 2012), pp.11-26.

${ }^{45}$ Ibid, p. 26.
} 
nuclear son muy improbables. Pero durante el largo tiempo que existan las armas nucleares, la OTAN será una alianza nuclear" ${ }^{\prime 4}$.

\section{La construcción de una nueva Estrategia Global europea, sin una política de control de la proliferación.}

A partir del año 2011 la Unión Europea debe hacer frente a un deterioro continuado de su entorno de seguridad que presenta graves incertidumbres. El estallido de la guerra en Libia y Siria y la pérdida de control de sus arsenales de armas biológicas y químicas ante la ausencia de mecanismos de control y verificación han incrementado la inestabilidad regional. La crisis de Ucrania ha repercutido en la pérdida de credibilidad de la Unión como actor capaz de desarrollar una política de seguridad y defensa operativa a largo plazo, especialmente en plena retirada de EE.UU. hacia el Pacífico ${ }^{47}$. El sistema de no proliferación internacional se encuentra al límite de su capacidad con el enfriamiento de las relaciones entre Rusia y EE.UU. que han implementado "programas masivos" 48 de modernización nuclear vaciando importantes avances realizados y el artículo 6 del tratado de no proliferación.

Las dinámicas que se están desarrollando en este nuevo contexto han alcanzado tal grado de complejidad que han dejado a la EES como un instrumento irrelevante, incapaz de hacer frente a los nuevos riesgos y amenazas ${ }^{49}$ con restricción de medios económicos y en pleno proceso de cuestionamiento de la integridad de la Unión potenciado por el efecto del Brexit. Durante este vacío estratégico que se produce entre la vigencia de una EES claramente obsoleta y la presentación de la Estrategia Global Europea en el año 2016, cuatro países europeos (España, Italia, Polonia y Suecia) solicitaron la redacción de un informe no oficial sobre el contenido tentativo de una Estrategia Europea a varios centros de estudio internacionales., que presentaron en el año 2013 el informe "Hacia una Estrategia Global Europea. Asegurando la influencia global europea en un mundo cambiante" Paradójicamente, el informe apenas prestaba atención al entorno de seguridad, no se centraba en riesgos o amenazas, aunque hablaba de instrumentos o líneas de acción, y presentaba una proyección global de carácter muy limitado, delimitada por una vaga declaración de principios y la priorización de aspectos casi exclusivamente económicos. La proliferación de ADM apenas se menciona en el documento.

La dinámica internacional del proceso de no proliferación nuclear se ha visto ralentizada a partir del año 2013 principalmente a causa del distanciamiento de las agendas nucleares de Rusia y EEUU. Mientras que el segundo ha mostrado cierto interés en continuar, según lo previsto por el tratado Nuevo START, con los objetivos de desarme nuclear, el primero ha centrado el foco de su atención sobre los sistemas de defensa anti-misiles, así como sobre el desarrollo de sistemas alternativos a las armas nucleares pero que puedan llevar a cabo el mismo rol estratégico que estas (como por ejemplo los vehículos aéreos no tripulados con capacidad ofensiva o la posibilidad de sustituir la carga nuclear de misiles

\footnotetext{
${ }^{46}$ Strategic Concept for the Defense and Security of the Members of the North Atlantic Treaty Organization, en http://www.nato.int/strategic-concept/pdf/Strat_Concept_web_en.pdf

47 Biscop, Sven: "The state of defence in Europe: dependence, deterrence and deployment", Global Affairs, Vol.1 No. 2 (2015), pp.169-182.

48 “It is 3 Minutes to Midnight”, The Bulletin of Atomic Scientis, en http://thebulletin.org/clock/2015

${ }^{49}$ European Political Strategy Centre. In Defence of Europe. Defence Integration as a Response to Europe's Strategic Moment. Issue 4/2015, June 2015, en https://ec.europa.eu/epsc/file/strategic-note-4-defence-europe_en

${ }^{50}$ Hacia una Estrategia Global Europea. Asegurando la influencia global europea en un mundo cambiante, en http://www.realinstitutoelcano.org/wps/portal/rielcano/contenido?WCM_GLOBAL_CONTEXT=/elcano/elcano _es/zonas_es/europa/european-global-strategy-final-report
} 
balísticos por cabezas convencionales modernas de similar efectividad) ${ }^{51}$. Además de este choque entre agendas, hay que tener en cuenta que ambos países, especialmente Rusia, han implementado o están realizando muy importantes programas de modernización de sus respectivos arsenales nucleares ${ }^{52}$.

A lo que se añadía las posibilidades crecientes de nuevos actores proliferadores. El Consejo de la UE constatará esta nueva situación en su reunión en Luxemburgo de 21 de octubre de 2013, afirmando que:

"The threat presented by the proliferation of weapons of mass destruction, as identified in the European Security Strategy of 2003, has not diminished and presents a growing risk to the EU's security environment. It is taking on new dimensions that represent challenges to which the EU must respond effectively: new communication tools allowing easier acquiring of sensitive knowledge and know-how by proliferators; new proliferation pathways; and the rapid development of science and technology, which facilitates the design of weapons of mass destruction". ${ }^{53}$

Por todo ello, teniendo en cuenta las posturas de los principales Estados nucleares, incluyendo el proceso de modernización iniciado por Reino Unido y Francia, anteriormente citado, el aumento en cantidad y capacidad de los arsenales de China, la escalada entre India y Pakistán, así como la situación de Corea del Norte, no resulta extraño que la Conferencia de revisión del TNP 2015, uno de cuyos ejes centrales era la creación de una zona libre de armas de destrucción masiva en Oriente Medio, según los acuerdos y previsiones establecidas en $1995^{54}$, fracasara. La Conferencia puso de manifiesto la delicada situación del proceso de no proliferación. Aunque el principal obstáculo fuese la situación de Oriente Medio, donde no se había avanzado, a pesar de la resolución de 1995 con motivo de la extensión indefinida del TNP, quedaron patentes los principales puntos de discordia: las diferencias entre países nuclearizados y no nuclearizados, la ausencia de medidas de desarme efectivas, las interpretaciones sobre la aplicación del artículo X del TNP y la situación de los protocolos adicionales relativos al desarrollo de tecnología nuclear para uso civil y la difusión de la misma $^{55}$.

Una cuestión importante para la política europea que dicha conferencia puso en evidencia, fue que, aunque se hubiera aprobado una posición común, las sensibilidades y posturas de los diversos Estados Miembros no son similares. Así, mientras que Austria e Irlanda mantienen, tradicionalmente, posturas "abolicionistas" y la mayoría de Estados Miembros de la UE se encuentran en una posición de equilibrio entre la no proliferación y el uso pacífico de la energía atómica, el Reino Unido se ha destacado siempre alineándose con el

\footnotetext{
${ }^{51}$ Pifer, Steven: "The future of U.S.-Russian arms control", 26 de febrero de 2016, en https://www.brookings.edu/research/the-future-of-u-s-russian-arms-control/

${ }^{52}$ Ibid.

53 “Council conclusions on ensuring the continued pursuit of an effective eu policy on the new challenges presented by the proliferation of weapons of mass destruction (WMD) and their delivery systems", Foreign Affairs Council meeting, Luxembourg, 21 October 2013, en http://www.consilium.europa.eu/uedocs/cms_data/docs/pressdata/EN/foraff/139067.pdf

${ }^{54}$ Salazar, op. cit. p. 162

${ }^{55}$ Wan,Wilred:"Why the 2015 NPT review conference fell apart", UNU-CPR, Centre for Policy Research, 28 de mayo de 2015, en https://cpr.unu.edu/why-the-2015-npt-review-conference-fell-apart.html
} 
bloque encabezado por EEUU ${ }^{56}$. También la postura de Francia, como país nuclear, ha creado problemas.

La publicación, en el año 2016, de la Estrategia Global para la Política Exterior y de Seguridad de la Unión Europea "Una visión común, una actuación conjunta: una Europa más fuerte" sigue la línea establecida por el informe de 2013 en materia de proliferación de ADM. En este caso la proliferación de ADM no se contempla como un elemento determinante en la seguridad de la Unión, aunque se menciona brevemente dentro de los órdenes regionales como uno de los ejes de cooperación con Magreb y Oriente Próximo ${ }^{57}$, América latina y el Caribe $^{58}$ y la península de Corea ${ }^{59}$.

La única otra mención a la no proliferación en la Estrategia se refiere a los esfuerzos de la Unión por favorecer la ampliación de la aplicación de las normas, los regímenes y las instituciones internacionales de gobernanza global ${ }^{60}$, únicamente en este contexto define la proliferación como una "amenaza creciente para Europa y el resto del mundo", Unión muestra su compromiso con la universalización de los tratados y regímenes multilaterales de no proliferación y de control de armamento ${ }^{62}$.

\section{Conclusiones.}

La Unión Europea no ha sido capaz de coordinar, de forma generalizada, las posiciones que permitan desarrollar una política de control de la proliferación creíble, con líneas de acción claras, objetivos concretos y priorizados y mecanismos específicos para alcanzarlos. Sin embargo, si ha contado con éxitos moderados en ámbitos de actuación concretos como en el control de exportaciones, incluido el material de doble uso.

La Estrategia Europea de Seguridad y la Estrategia contra la proliferación de armas nucleares y sus vectores han mantenido una ambigüedad calculada fruto de la dificultad de la Unión en la consecución del consenso, optando por el alineamiento con las iniciativas de no proliferación existentes en la comunidad internacional, en vez de articular una política de control de la proliferación propia, y que se resumía en continuar apoyando un multilateralismo efectivo, estrecha cooperación con socios fundamentales y con otros Estados terceros, y una utilización efectiva y complementaria de todos los instrumentos disponibles y recursos financieros. ${ }^{63}$

La Unión ha sido incapaz de desarrollar una política de control de la proliferación proactiva con una visión global y se ha dejado llevar por el efecto de arrastre de las crisis coyunturales de seguridad que se han ido produciendo ${ }^{64}$. Esta situación se "oficializa" en la

\footnotetext{
${ }^{56}$ Salazar, op. cit. p. 161

${ }^{57}$ Estrategia global para la politica exterior y de seguridad de la Unión Europea "Una visión común, una actuación conjunta: una Europa más fuerte, p. 27, en https://eeas.europa.eu/top_stories/pdf/eugs_es_pdf ${ }^{58}$ Ibid., p. 29.

${ }^{59}$ Ibid., p. 30.

${ }^{60}$ Ibid., p. 33.

${ }^{61}$ Ibid., p. 33.

${ }^{62}$ La Estrategia da continuidad a las líneas de acción anteriores constatando que la UE "participará activamente en los regímenes de control de las exportaciones, reforzará las normas comunes por las que se rigen las políticas de los Estados miembros en materia de exportación de material y tecnologías militares, incluido el material de doble uso, y apoyará a las autoridades de control de las exportaciones en terceros países y a los organismos técnicos encargados de mantener los regímenes de control de armamento".

63 "The fight against proliferation of WMD", European Union External Action, en http://eeas.europa.eu/nonproliferation-and-disarmament/wmd/index_en.htm

${ }^{64}$ Villarino, Camilo. (2009): Un mundo en cambio, Madrid. Icaria, p. 90.
} 
estrategia de 2016 constatando que la UE empleará "todos los medios a nuestro alcance para contribuir a la resolución de crisis de proliferación, como ya hicimos de forma fructífera en el caso del programa nuclear iraní" 65 .

A partir del año 2013 podemos observar un cambio de filosofía en el tratamiento de la proliferación por parte de los documentos claves de la Unión. Si antes la proliferación de ADM era "la mayor amenaza potencial" a la seguridad europea, ahora se ha producido una dilución en la percepción de la amenaza que choca frontalmente con el contexto actual de seguridad francamente deteriorado después del fracaso de la Conferencia de revisión del TNP de 2015. El Bulletin of the Atomic Scientist resume así la situación del año 2016:

"A pesar de que el acuerdo de Irán estaba siendo finalizado, las tensiones entre Estados Unidos y Rusia se elevaron a niveles que recuerdan a los peores momentos de la Guerra Fría. Los conflictos en Ucrania y Siria continuaban, acompañados de bravatas peligrosas y arriesgadas, mientras Turquía, un miembro de la OTAN, derribó un avión de combate ruso involucrado en Siria, el director de una agencia de noticias estatal rusa realizó declaraciones acerca de convertir a Estados Unidos en ceniza radiactiva, y la OTAN y Rusia reposicionamiento recursos militares para la realización de ejercicios significativos. Washington y Moscú siguen adhiriéndose a la mayoría de los acuerdos de control de armas nucleares existentes, pero los Estados Unidos, Rusia, y otros países con armas nucleares se han involucrado en la modernización de sus arsenales nucleares, lo que sugiere que tienen previsto mantener y conservar la inmediata disposición de las armas nucleares durante décadas, al menos - a pesar de sus promesas, codificadas en el Tratado de no proliferación nuclear, de procurar el desarme nuclear" ${ }^{66}$.

La nueva estrategia de 2016 plantea la cuestión de la no proliferación de ADM sólo de forma tangencial, sin abordar el problema directamente, un problema fundamental en la seguridad. Esto supone un claro paso atrás para la seguridad común europea, que ha optado por una solución de mínimos que pone fin a los avances relativos que se habían realizado en la materia. Las dificultades de la Unión a la hora de articular una política proactiva en este ámbito han sido cercenadas por la imposibilidad de alcanzar un cierto consenso sobre el camino a seguir, derivada, ante todo, de la existencia de dos Estados con armas nucleares en su seno. El nuevo documento es un claro testimonio de la situación de progresivo abandono que este tema padece en la política de la Unión, y al que probablemente se verá abocado en el futuro próximo, aunque esta amenaza es prioritaria.

El Brexit plantea algunas cuestiones que de momento tienen difícil respuesta. No obstante, parece seguro predecir que un Reino Unido fuera de la Unión Europea, sin el "lastre" de las posturas comunitarias, continuará por el camino de la modernización de sus arsenales, lo cual es improbable que contribuya a que la región sea percibida como más segura. Por otro lado, la Unión sin el Reino Unido es probable que pueda ofrecer una postura común mucho más consensuada y sólida en esta materia, aunque el peso de Francia sin duda contribuirá a la tibieza de cualquier política futura.

\footnotetext{
${ }^{65}$ Estrategia global para la politica exterior y de seguridad de la Unión Europea "Una visión común, una actuación conjunta: una Europa más fuerte, p. 27, en https:/leeas.europa.eu/top_stories/pdf/eugs_es_.pdf ${ }^{66}$ Doomsday Clock hands remain unchanged, despite Iran deal and Paris talks, en http://thebulletin.org/pressrelease/doomsday-clock-hands-remain-unchanged-despite-iran-deal-and-paris-talks9122
} 


\section{Bibliografía Seleccionada}

1995 Review and Extension Conference of the Parties to the Treaty on the Non-Proliferation of Nuclear Weapons 17 April to 12 May 1995 - New York, en http://www.un.org/Depts/ddar/nptconf/162.htm

2000 Review Conference of the Parties to the Treaty on the Non-Proliferation of Nuclear Weapons 24 April - 19 May 2000, New York, en https://www.un.org/disarmament/wmd/nuclear/npt2000/

2005 Review Conference of the Parties to the Treaty on the Non-Proliferation of Nuclear Weapons 2 - 27 May 2005, New York, en http://www.un.org/en/conf/npt/2005/reports.html

2010 Review Conference of the Parties to the Treaty on the Non-Proliferation of Nuclear Weapons 3 - 28 May 2010, New York, en http://www.un.org/en/conf/npt/2010/

2015 Review Conference of the Parties to the Treaty on the Non-Proliferation of Nuclear Weapons 27 April - 22 May 2015 , New York, en http://www.un.org/en/conf/npt/2015/

Álvarez, Milagros: "La Aproximación de las Estrategias de la UE y de los EEUU en la lucha contra la proliferación de armas de destrucción masiva", UNISCI Discussion Papers, n 30 (octubre 2012)

Armas de destrucción masiva: lucha contra la proliferación, en http://eur-lex.europa.eu/legalcontent/ES/TXT/?uri=uriserv\%3Al33234

Biscop, Sven: "The state of defence in Europe: dependence, deterrence and deployment", Global Affairs, Vol.1 No. 2 (2015), pp.169-182.

Council Conclusions and new lines for action by the European Union in combating the proliferation of weapons of mass destruction and their delivery systems. European Union External Action The fight against proliferation of $W M D$, en http://eeas.europa.eu/non-proliferation-and-disarmament/wmd/index en.htm

Council conclusions on ensuring the continued pursuit of an effective eu policy on the new challenges presented by the proliferation of weapons of mass destruction (WMD) and their delivery systems, Foreign Affairs Council meeting, Luxembourg, 21 October 2013, en http://www.consilium.europa.eu/uedocs/cms_data/docs/pressdata/EN/foraff/139067.pdf

Declaración del presidente del Consejo de Seguridad de la ONU de 31 de enero de 1992, S/23500, en http://www.securitycouncilreport.org/un-documents/document/PKO\%20S\%2023500.php, p. 3

Dee Megan: "Explaining European Union performance in the nuclear non-proliferation treaty review Conference: Limited ambitions but pragmatic positioning”, UNISCI Discussion Papers, nº 30 (Octubre 2012)

Discurso del Presidente de los Estados Unidos, Barack Obama en Praga. 5 abril 2009, en http://npsglobal.org/esp/discursos/574-discurso-de-barack-obama-en-praga.html

Doomsday Clock hands remain unchanged, despite Iran deal and Paris talks, en http://thebulletin.org/pressrelease/doomsday-clock-hands-remain-unchanged-despite-iran-deal-and-paris-talks9122

European Political Strategy Centre. In Defence of Europe. Defence Integration as a Response to Europe's Strategic Moment. Issue 4/2015, June 2015, en https://ec.europa.eu/epsc/file/strategic-note-4-defence-europe_en Estrategia de la UE contra la proliferación de armas de destrucción masiva, en http://register.consilium.europa.eu/doc/srv?l=ES\&f=ST\%2015708\%202003\%20INIT

Fact Sheet on U.S. Missile Defense Policy. A Phased, Adaptive Approach for Missile Defense in Europe, 17 Sept. 2009), en https://www.whitehouse.gov/the-press-office/fact-sheet-us-missile-defense-policy-a-phasedadaptive-approach-missile-defense-eur

Garrido Rebolledo, Vicente: "Redefinición y respuesta de los europeos frente a las nuevas amenazas: proliferación de armamento de destrucción masiva", en La seguridad europea y las incertidumbres del 11 de septiembre, Monografías del CESEDEN nº 61, mayo 2003 
Guisández Gómez, Javier: "Iniciativas multilaterales de no proliferación", en Respuestas al reto de la proliferación, Documentos de Seguridad y Defensa, CESEDEN, $n^{\circ}$ 27, marzo 2009

Hacia una Estrategia Global Europea. Asegurando la influencia global europea en un mundo cambiante, en http://www.realinstitutoelcano.org/wps/portal/rielcano/contenido?WCM GLOBAL CONTEXT=/elcano/elcano es/zonas es/europa/european-global-strategy-final-report

Hidalgo Garcia, M. ${ }^{a}$ del Mar (2011): "Iniciativas para la lucha contra la proliferación de las armas de destrucción masiva", en Proliferación de ADM y de tecnología avanzada, Ministerio de Defensa. Cuadernos de estrategia n ${ }^{\circ}$ 153. IEEE (septiembre 2011).

Informe sobre la aplicación de la Estrategia Europea de Seguridad - Ofrecer seguridad en un mundo en evolución, Bruselas, 11 de diciembre de 2008, en http://www.consilium.europa.eu/uedocs/cms data/docs/pressdata/ES/reports/104637.pdf

Miasnikov, Eugene: "Modernizing nuclear arsenals: Whether and how" y "Modernizing and "zero": compatible tendencies?", Bulletin of Atomic Scientists, (enero 2015), en http://thebulletin.org/modernizing-nuclear-arsenalswhether-and-how7881

Nia, Mahdi Mohammad: "Counter-proliferation vs Non-proliferation.", PA Pundits-International, 2008, en https://papundits.wordpress.com/2008/11/20/counter-proliferation-vs-non-proliferation/

NTI. France, en http://www.nti.org/learn/countries/france/nuclear/

NTI. United Kingdom, en http://www.nti.org/learn/countries/united-kingdom/

Pifer, Steven: "The future of U.S.-Russian arms control", 26 de febrero de 2016, en https://www.brookings.edu/research/the-future-of-u-s-russian-arms-control/

Portela, Clara: "The Role of the EU in the Non-Proliferation of Nuclear Weapons: The Way to Thessaloniki and Beyond", Peace Research Institute Frankfurt, PRIF Reports, n65 (2003), p. 29.

Proliferation Security Initiative: Statement of Interdiction Principles, en http://www.psionline.info/Vertretung/psi/en/07-statement/Interdiction-Principes.html

Salazar, Gonzalo de: "El tratado de no proliferación de armas nucleares: los temas clave en la conferencia de examen en 2015", UNISCI Discussion Papers, $\mathrm{n}^{\circ} 38$ (mayo 2015), p. 158.

Shared Vision, Common Action: A Stronger Europe. A Global Strategy for the European Union's Foreign and Security Policy, en https://eeas.europa.eu/top_stories/pdf/eugs_review_web.pdf

Strategic Concept for the Defense and Security of the Members of the North Atlantic Treaty Organization, en http://www.nato.int/strategic-concept/pdf/Strat_Concept_web_en.pdf

“The fight against proliferation of WMD”, European Union External Action, en http://eeas.europa.eu/nonproliferation-and-disarmament/wmd/index_en.htm

The National Security Strategy of the United Status of America (NSS). Washington D.C., September 2002, en http://www.whitehouse.gov/nsc/nss.html.

Towards a European Global Strategy. Securing European influence in a changing world, May 13, 2013, en http://www.realinstitutoelcano.org/wps/wcm/connect/4c2675804fc8b86b80b5caccba746acc/EGS_Report.pdf?M $\mathrm{OD}=\mathrm{AJPERES} \& \mathrm{CACHEID}=4 \mathrm{c} 2675804 \mathrm{fc} 8 \mathrm{~b} 86 \mathrm{~b} 80 \mathrm{~b} 5 \mathrm{caccba} 746 \mathrm{acc}$

Una Europa segura en un mundo mejor - Estrategia Europea de Seguridad 2003, Bruselas 12 de diciembre de 2003.en https://www.consilium.europa.eu/uedocs/cmsUpload/031208ESSIIES.pdf

Wan,Wilred:"Why the 2015 NPT review conference fell apart", UNU-CPR, Centre for Policy Research, 28 de mayo de 2015, en https://cpr.unu.edu/why-the-2015-npt-review-conference-fell-apart.html 